\title{
BMJ Open Solution Focused Brief Therapy in Post- Stroke Aphasia (SOFIA): feasibility and acceptability results of a feasibility randomised wait-list controlled trial
}

To cite: Northcott $S$,

Thomas S, James K, et al. Solution Focused Brief Therapy in Post-Stroke Aphasia (SOFIA): feasibility and acceptability results of a feasibility randomised waitlist controlled trial. BMJ Open 2021;11:e050308. doi:10.1136/ bmjopen-2021-050308

- Prepublication history and additional supplemental material for this paper are available online. To view these files, please visit the journal online. (http://dx.doi.org/10.1136/ bmjopen-2021-050308)

Received 16 February 2021 Accepted 14 July 2021

Check for updates

(C) Author(s) (or their employer(s)) 2021. Re-use permitted under CC BY-NC. No commercial re-use. See rights and permissions. Published by BMJ.

For numbered affiliations see end of article.

Correspondence to

Dr Sarah Northcott;

Sarah.northcott@city.ac.uk

\section{ABSTRACT}

Objectives The Solution Focused Brief Therapy in PostStroke Aphasia feasibility trial had four primary aims: to assess (1) acceptability of the intervention to people with aphasia, including severe aphasia, (2) feasibility of recruitment and retention, (3) acceptability of research procedures and outcome measures, and (4) feasibility of delivering the intervention by speech and language therapists.

Design Two-group randomised controlled feasibility trial with wait-list design, blinded outcome assessors and nested qualitative research.

Setting Participants identified via two community NHS Speech and Language Therapy London services and through community routes (eg, voluntary-sector stroke groups).

Participants People with aphasia at least 6 months post stroke.

Intervention Solution-focused brief therapy, a psychological intervention, adapted to be linguistically accessible.

Participants offered up to six sessions over 3 months, either immediately postrandomisation or after a delay of 6 months. Outcome measures Primary endpoints related to feasibility and acceptability. Clinical outcomes were collected at baseline, 3 and 6 months postrandomisation, and at 9 months (wait-list group only). The candidate primary outcome measure was the Warwick-Edinburgh Mental Well-being Scale. Participants and therapists also took part in in-depth interviews.

Results Thirty-two participants were recruited, including 43.8\% with severe aphasia. Acceptability endpoints: therapy was perceived as valuable and acceptable by both participants ( $\mathrm{n}=30$ interviews) and therapists ( $n=3$ interviews); $93.8 \%$ of participants had $\geq 2$ therapy sessions ( $90.6 \%$ had $6 / 6$ sessions). Feasibility endpoints: recruitment target was reached within the prespecified 13-month recruitment window; $82.1 \%$ of eligible participants consented; $96.9 \%$ were followed up at 6 months; missing data $<0.01 \%$. All five prespecified feasibility progression criteria were met.

Conclusion The high retention and adherence rates, alongside the qualitative data, suggest the study design was feasible and therapy approach acceptable even to people with severe aphasia. These results indicate a definitive randomised controlled trial of the intervention would be feasible.
Strengths and limitations of this study

- People with different presentations of aphasia, including severe aphasia, were enabled to participate in a psychological therapy trial.

- People with aphasia were involved in designing the trial and gave ongoing advice throughout.

- Both qualitative and quantitative approaches were used to answer questions around feasibility and acceptability.

- The trial investigated the feasibility of speech and language therapists delivering the intervention and explored their perspectives on what training and support was useful.

- A weakness of the trial is that family members were not recruited, so it was not possible to capture their perspective on the intervention or study processes.

Trial registration number NCT03245060.

\section{INTRODUCTION}

Around one-third of people who have a stroke will experience aphasia, a language disability. ${ }^{1}$ Living with poststroke aphasia places someone at risk of having a reduced social network ${ }^{2}$ and developing depression. ${ }^{3}$ Having a stroke and aphasia has been described as a traumatic and distressing event ${ }^{4}$ and can profoundly challenge a person's identity and life plans. ${ }^{5}$ Despite people with aphasia being at greater risk of adverse psychosocial consequences compared with those who do not have aphasia post stroke, ${ }^{6} 7$ they are often excluded from psychological stroke research. ${ }^{8}$ This is slowly changing: there has been research, for example, showing the effectiveness of behavioural activation therapy delivered by assistant psychologists, ${ }^{9}$ and recent feasibility trials reporting promising results for a singing intervention ${ }^{10}$ and peer befriending. ${ }^{11}$ Nonetheless, a review concluded that there is still limited evidence 
for effective psychological therapies for this client group, ${ }^{12}$ including limited research exploring the role of speech and language therapists (SLTs) in delivering a psychological therapy. Further, it is a concern that people with aphasia, particularly more severe aphasia, are reported not to have equitable access to mental health services. ${ }^{1314}$ There are challenges in adapting language-based psychological therapies for people with a language disability. As such, there is a need to investigate psychological therapies that are accessible and effective for people with poststroke aphasia.

A promising psychological intervention to enhance well-being is solution-focused brief therapy (SFBT), which focuses on a client's expertise and resources to support them in moving forward in living well with their health condition. ${ }^{15}$ It has been shown to be flexible enough to be adapted for client groups who have limited language, such as those with an intellectual disability. ${ }^{16} \mathrm{~A}$ recent meta-analysis found a small to medium effect size in achieving positive psychosocial outcomes when used in medical settings $(d=0.34, \mathrm{p}<0.05) .{ }^{17}$

A randomised controlled trial of working-age people post stroke, excluding people with aphasia, found receiving 10 sessions of SFBT resulted in significantly better mood and lower anxiety compared with usual care. ${ }^{18}$ An initial proof-of-concept study explored an aphasia-accessible version of SFBT with people who had mild-moderate chronic aphasia: participants reported finding the approach highly acceptable, and there were promising trends in terms of improved mood and participation. ${ }^{19}$ The Solution Focused Brief Therapy in PostStroke Aphasia (SOFIA) trial ${ }^{20}$ builds on this preliminary work.

The main aim of the SOFIA trial was to assess the feasibility of conducting a future definitive trial investigating the clinical and cost effectiveness of SFBT for people living with aphasia. An in-depth exploration of participants' experiences of the therapy, ${ }^{21}$ treatment fidelity results and a pilot economic evaluation, including analysis of the European Quality of Life Scale, are reported elsewhere. This paper reports on the primary objectives of the trial, which were to evaluate the acceptability of the intervention, the feasibility of recruitment and retention, the acceptability of research procedures, and the the feasibility of SLTs delivering the intervention.

\section{METHODS}

SOFIA was a single-blind randomised wait-list controlled feasibility trial comparing SFBT plus usual care to usual care alone for people living with poststroke aphasia. Full details are provided in the protocol paper. ${ }^{20}$ All participants completed baseline assessments and were then randomised to receive the intervention either immediately or after a delay of 6 months. Assessments were completed at 3 and 6 months postrandomisation, and at 9 months for the wait-list group only. All participants were invited to take part in in-depth interviews at 6 months; the wait-list group was additionally interviewed at 9 months. Therapists and local collaborators at National Health Service (NHS) sites were also interviewed. Recruitment took place between October 2017 and November 2018. There were no changes to the trial design during the trial. The trial was registered with ClinicalTrials.gov on 10 August 2017, based on protocol V.2 (24 August 2017, doi. org/10.25383/city.8053415.v1). Primary and secondary outcome measures reported are consistent with the protocol and trial registry.

The study design and reporting has been guided by the Consolidated Standards of Reporting Trials (CONSORT) 2010 extension statement to randomised pilot and feasibility trials. ${ }^{22}$ Qualitative data have been reported according to the Standards for Reporting Qualitative Research Guidelines. ${ }^{23}$ Qualitative results are reported in online supplemental appendix material 1 and summarised in the main text. Identifying details have been removed.

\section{Patient and public involvement}

An initial consultation event with people with aphasia and a carer advised on the study aims and design, including choice of control condition. Subsequently, the SOFIA Aphasia Advisory Group has supported decision making on recruitment processes, eligibility criteria, project information documents and consent forms, choice and presentation of outcome measures, interview topic guides, and dissemination strategies. For example, they advocated for the inclusion of people with severe aphasia, advised on how to make study processes a positive experience for participants and supported creating a project blog.

\section{Participants}

\section{Setting}

Participants were identified through two London NHS community SLT services; and through non-NHS community sources (eg, voluntary-sector groups). Visits were conducted at the participants' choice of venue, most commonly their home or university clinic.

\section{Eligibility criteria}

Participants were eligible if they had a diagnosis of ischaemic or haemorrhagic stroke and were at least 6 months post stroke, aged 18 or older and had aphasia as determined by an SLT. Exclusion criteria comprised other diagnoses affecting cognition such as dementia, severe uncorrected visual or hearing problems, severe or potentially terminal comorbidity, being a non-fluent English speaker prior to the stroke (based on self or family report), receiving a psychological or psychiatric intervention at the time of recruitment and not having mental capacity. People with any severity of aphasia were included so long as they retained capacity. Use of antidepressants or rehabilitation therapy was not a reason for exclusion, nor were people excluded based on their depression or well-being scores. 
Recruitment and consent processes

SLTs and group coordinators linked to SLT services at participating sites screened people on their caseloads and gained consent to pass on contact details to the research team. For those participants recruited via community, eligibility was checked through relying on self-report. The principal investigator, an experienced SLT, met all potential participants face-to-face to discuss the project and enabled them to make an informed choice. All information sheets and consent forms were designed to be accessible to people with aphasia (eg, using short simple sentences and suitable pictorial images to support key concepts). Written informed consent was obtained from all participants.

\section{Intervention description}

The intervention, therapist training and supervision, treatment fidelity measures, and therapy manual are described in the SOFIA TIDieR checklist (https:// city.figshare.com/articles/SOFIA_TIDieR_checklist/ 8058539). SFBT explores a person's resources and hopes for the future. Rather than focusing on deficits or diagnosing problems, it invites the client to notice instances of success and what is already working, using these as building blocks in moving forward. Within the SOFIA trial, SFBT was adapted to be linguistically accessible and emphasis was placed on acknowledging distress. SFBT was designed to be a brief intervention, typically three to five sessions, with the client taking ownership of the ending of the therapy. ${ }^{24} 25$ Preliminary work suggested that people with aphasia were likely to benefit from more sessions, as their language disability means it can take longer to cover typical SFBT therapy activities. ${ }^{19}$ SOFIA participants were therefore offered up to six sessions and were invited to choose how they spaced their sessions over a 3-month period and to have as many of the six sessions as they perceived would be useful to them. Participants were offered the therapy either immediately postrandomisation or after a delay of 6 months. Both groups received all usual care, including healthcare, social care and voluntary services. The trial intervention was provided by SLTs, who received specialist training, regular supervision and additional real-time support as needed.

\section{Outcomes}

The main endpoints related to acceptability and feasibility objectives. Five prespecified criteria were used to assess the feasibility of progressing to a definitive trial, interpreted alongside the qualitative findings. In addition, adverse events were recorded. Aphasia was assessed at baseline using the Frenchay Aphasia Screening Test (FAST). ${ }^{26}$

\section{Primary endpoints}

1. Acceptability of the intervention to participants and trial clinicians, based on adherence rates, scores on the Session Rating Scale (SRS) assessing therapeutic alliance, interviews with participants and clinicians.
Prespecified criterion (1): proportion of participants who adhered (had at least two therapy sessions) at least $80 \%$.

2. Feasibility of recruitment and retention to the trial, based on the proportion who gave permission for their contact details to be passed to the research team, proportion who consented, number of randomised each month and attrition rates. Prespecified criterion (2): proportion of eligible participants who consented at least $60 \%$. Prespecified criterion (3): proportion of participants who were followed up at 6 months postrandomisation at least $70 \%$.

3. Acceptability of research procedures and outcome measures, based on participant interviews, drop-out rates and rates of missing data. Prespecified criterion (4): proportion of missing data per scale less than $15 \%$ for participants with mild-moderate aphasia. Prespecified criterion (5): proportion of all participants who completed the Depression Intensity Scale Circles (DISCs) at least $90 \%$.

4. Feasibility of delivering the intervention by SLTs, based on clinician and participant interviews.

\section{Patient-reported outcome measures}

At baseline and at 3 and 6 months postrandomisation (plus 9 months, wait-list group only), outcomes likely to be used in a future definitive trial were measured face-toface. The primary clinical outcome was psychological wellbeing, measured using the Warwick-Edinburgh Mental Well-being Scale (WEMWBS), ${ }^{27}$ with primary comparison point of 6 months. Secondary outcomes were General Health Questionnaire-12 (GHQ-12), ${ }^{28}$ measuring mood, both overall score (range $0-12$ ) and as a categorical variable (participants scoring $<3$ classified as having no or low distress); DISCs, ${ }^{29}$ also measuring mood; and Communicative Participation Item Bank (CPIB) ${ }^{30}$ measuring participation. The SRS, ${ }^{31}$ measuring therapeutic alliance, was completed during the in-depth interview.

\section{Sample size}

The planned sample size was 32 participants, 16 in each arm. This was considered sufficient to estimate parameters for a larger trial, such as recruitment and retention rates, with acceptable precision. ${ }^{32-34}$

\section{Randomisation and blinding}

Participants were randomised 1:1 using minimisation with a random component via an independent randomisation service at King's Clinical Trials Unit. Minimisation stratification factors were: site and aphasia severity (mild-moderate or severe). Participants who scored $<7$ on either receptive or expressive domains of the FAST were classified as having severe aphasia. Outcome assessors were blind to group allocation; and as the 9-month assessment applied only to the wait-list group, assessors were blind to both group allocation and timepoint for 9-month assessment. Participants, trial clinicians, the qualitative 
researchers, statistician, and the principal investigator were aware of group allocation.

\section{Analytical methods}

A CONSORT diagram was used to display recruitment and adherence. Feasibility outcomes were estimated, along with 95\% CIs, using the binomial exact distribution for proportions, and Poisson for rates. Descriptive statistics for potential trial outcomes were summarised for the entire trial population and by trial arm at all timepoints. Missing data were summarised at item, scale and administration levels.

Mean differences in outcomes between groups at 6 months postrandomisation and 95\% CI were estimated using linear mixed models with maximum likelihood estimation with a random effect for participants to account for repeated measures of the outcome data. Dependent variables were post-treatment outcome measures at 3 and 6 months. Fixed effects comprised: baseline measure of the outcome; trial arm; randomisation stratifiers (site and aphasia severity); dummy variable for timepoint; dummy variables for therapist; a trial arm $\times$ timepoint interaction term; and time post onset of stroke, rehabilitation status, gender and age as these were clinically judged post hoc to be potentially imbalanced at baseline. Effect sizes were calculated by dividing mean differences by baseline SDs over the whole sample for each outcome to allow trial arm comparison between measures and timepoints. The two arms were compared on dichotomised GHQ-12 caseness (high distress $\geq 3$, low distress 0-2) using ORs estimated from mixed effect logistic regression using the same fixed and random effects as the linear models. In addition, as a prespecified exploratory analysis, the analysis was rerun excluding participants with high well-being $(\geq 59 / 70$ on the WEMWBS) at baseline. Descriptive statistics over time for people with severe versus mild-moderate aphasia were also calculated. Since the intervention was therapist led, WEMWBS intracluster correlation coefficient (ICC) was estimated for participants treated by the same therapist for outcomes 6 months postrandomisation using a linear mixed effects model with the 6-month WEMWBS as the dependent variable and a random intercept for therapist.

All statistical analyses were conducted using Stata V.16. Qualitative data were transcribed verbatim and analysed using framework analysis ${ }^{35}$; further details of the qualitative analysis are provided in online supplemental appendix 1.

\section{RESULTS}

Figure 1 CONSORT diagram shows participants' journeys through the study. Out of the 54 who were identified, 39 were eligible. The most common reason for ineligibility was lacking capacity $(n=5)$. Of those who were eligible, seven declined to consent into the trial, most commonly because they preferred no further input $(n=5)$.

All participants who received the intervention $(n=30)$ also took part in in-depth interviews post intervention. In addition, 13 participants from the wait-list group took part in interviews 6 months postrandomisation (preintervention).
Finally, all three trial clinicians and both local collaborators at the NHS participant identification centres participated in in-depth interviews at the end of the trial.

\section{Participant characteristics}

Table 1 displays baseline characteristics of participants overall and by trial arm. The average age was 67.2 years (SD 12.9 years), and average time poststroke was 33.9 months (SD 33.3). The majority were white $(68.8 \%)$, living with a spouse or partner $(53.1 \%)$, and had mildmoderate aphasia (56.3\%). Exactly half were female. The trial arms were potentially imbalanced at baseline with the wait-list group on average being fewer months poststroke (22.5 months vs 43.9 months), more likely to be receiving rehabilitation $(60 \%$ vs $29.4 \%)$, older $(71.3$ years vs 63.5 years) and more likely to be female ( $66.7 \%$ vs $35.3 \%)$.

\section{Feasibility and acceptability results}

Feasibility and acceptability parameters are presented in table 2. All five prespecified progression criteria were satisfied. Main qualitative findings are reported briefly as follows; a full thematic analysis is provided in online supplemental appendix 1 .

Primary endpoint 1: acceptability of the intervention to participants and trial clinicians

The proportion of participants who adhered (had at least two therapy sessions) across both groups was 93.8\%: 29/32 participants had the maximum six sessions; 1 participant had five sessions; 2 withdrew. Scores on the SRS also indicated high levels of acceptability (table 2 ).

\section{Participant and therapist perspective on therapy}

Participants $(n=30)$ were unanimous in finding the therapy approach acceptable. ${ }^{21}$ Trial clinicians $(n=3)$ found the intervention rewarding, meaningful and energising to deliver.

It's the most satisfying kind of therapy I've ever done really, yeah, it's, you feel like you've really helped someone in quite a profound way sometimes, so that's, that's a really nice feeling. (Trial clinician)

While aspects of the therapy needed to be modified to be more linguistically accessible, the underlying assumptions of the approach were perceived to work well for people with aphasia, including severe aphasia. It was, however, sometimes challenging to find a focus for the therapy when participants were already content with their lives or wanted therapy tasks to promote language recovery.

Primary endpoint 2: feasibility of recruitment and retention to the trial

It was feasible to recruit to target and on time: 32 participants were recruited within the prespecified 13-month time window. The two strongest routes for recruitment were longer-term NHS-funded therapy groups and support services $(37.5 \%)$ and referrals from NHS SLT community caseloads $(28.1 \%)$. The proportion of eligible 


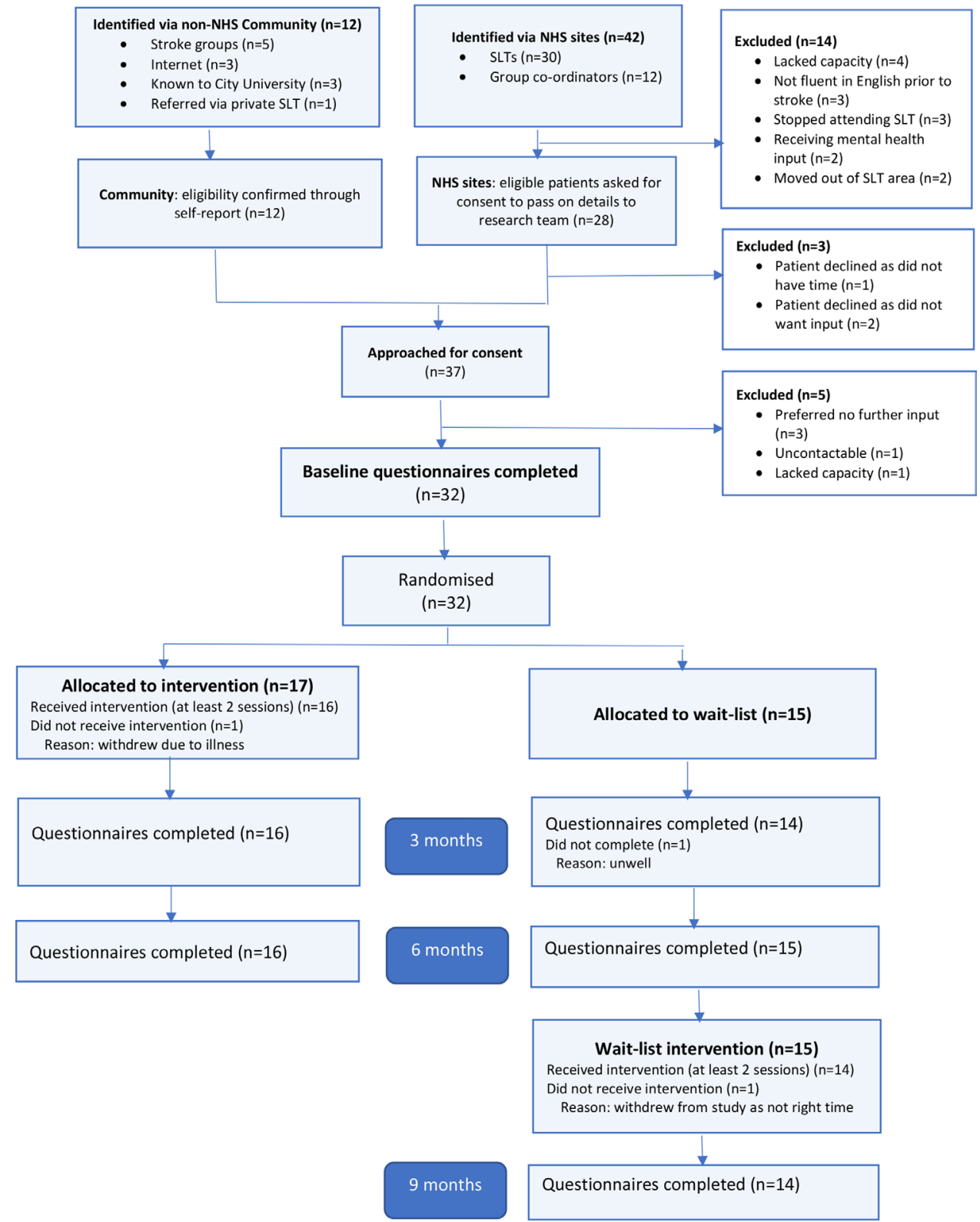

Figure 1 Solution Focused Brief Therapy in Post-Stroke Aphasia trial Consolidated Standards of Reporting Trials diagram. SLT, speech and language therapist.

participants who consented was $82.1 \%$; the proportion who were followed up at 6 months postrandomisation was 96.9\%. Only two participants withdrew due to ill-health and it being 'not the right time'.

\section{Local collaborator perspective on recruitment}

The local collaborators were positive about their involvement in the project. They identified six main facilitators to successful recruitment: (1) initial site visit, which generated enthusiasm and meant there was a 'face to the project'; (2) easy channels for ongoing contact with the principal investigator; (3) the principal investigator being approachable and flexible, creating 'a feeling of partnership'; (4) working with local systems and staff preferences wherever possible; (5) a local champion to advocate for the project, as well as buy-in from senior management; and (6) enthusiasm from team members due to their belief in the study, the inclusion of people with severe aphasia and being able to offer the study intervention at the point of discharge. The main challenges were work pressures, compounded by high turnover of staff, information technology systems and fewer than expected eligible patients. For one site, there was also anxiety around the wait-list design. Both local collaborators considered there were advantages in SLTs identifying potential participants as they were skilled at facilitating the communication of someone with aphasia. The sites were excited to be part of something 'bigger' and innovative; it helped raise the profile of their SLT service; and they were highly positive about the training (both sites offered training in the study intervention at the end of the trial). 
Table 1 Summary of baseline characteristics by treatment group and overall

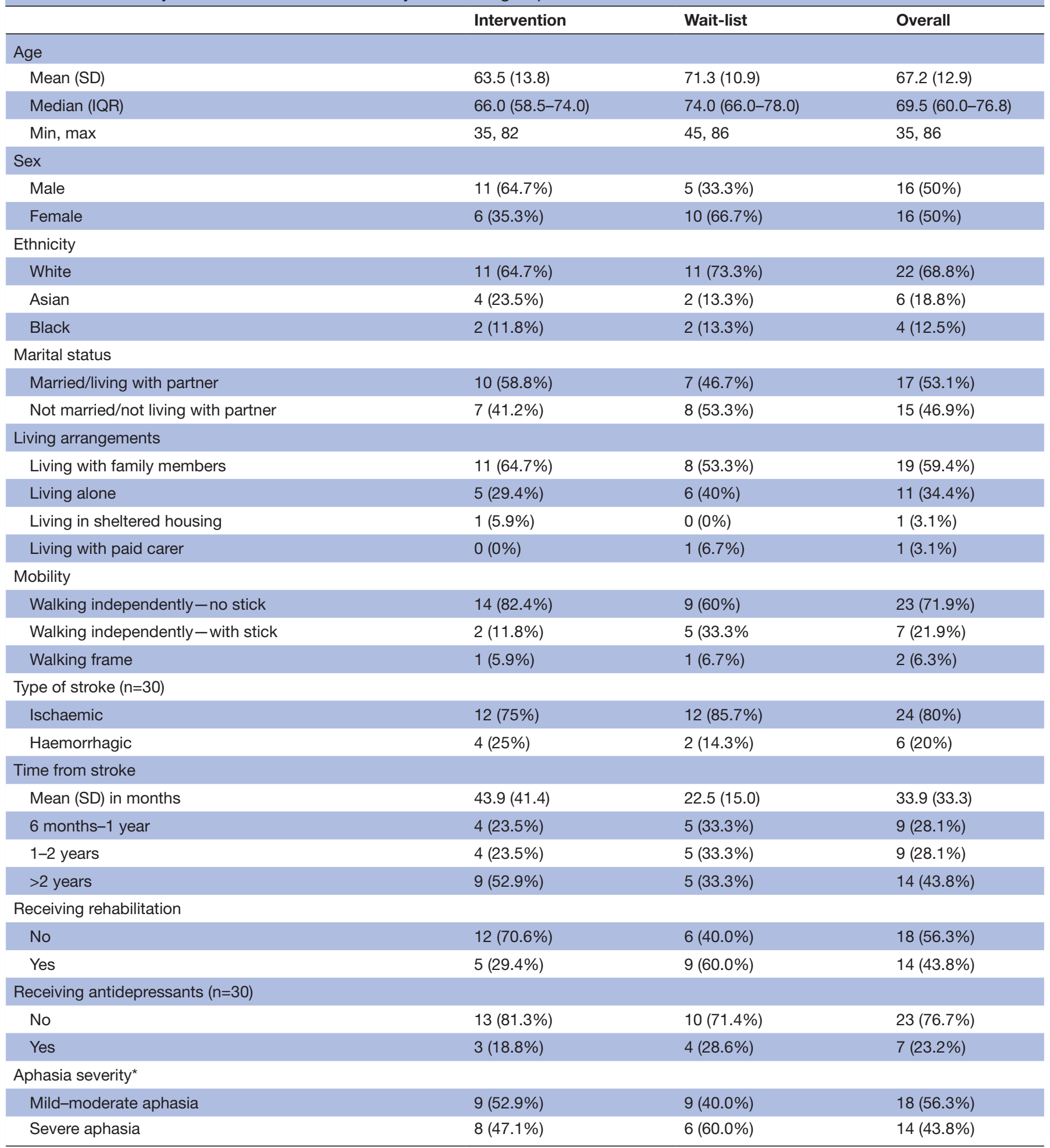

${ }^{*}$ Classified as having severe aphasia if scored $<7 / 15$ on the receptive and/or expressive domains of the Frenchay Aphasia Screening Test (FAST).

The team have really enjoyed being part of this project, I think they found it really valuable, and they really enjoyed the training session... it's so nice to see them enthusiastic and really proud of a piece of work they've done. (Local collaborator)

\section{Participant perspective on recruitment and retention}

Participants with aphasia took part in the study to help others, for companionship, out of curiosity and to improve their language. Three main factors explained why they continued: they enjoyed the research visits, perceived the 
Table 2 Feasibility and acceptability parameters

\begin{tabular}{|c|c|c|}
\hline Parameter & Proportion $(95 \% \mathrm{Cl}) /$ rate & Numbers used \\
\hline \multicolumn{3}{|l|}{ Endpoint 1: acceptability of therapy } \\
\hline $\begin{array}{l}\text { Criterion } 1 \text { met: proportion who adhered (had at least two therapy } \\
\text { sessions) (\%) }\end{array}$ & 93.8 (79.2 to 99.2$)$ & $30 / 32$ \\
\hline Proportion who had all six therapy sessions (\%) & 90.6 (75.0 to 98.0$)$ & $29 / 32$ \\
\hline Acceptability: Session Rating Scale (SRS) & Mean (SD): $36.1(4.5)$ & $\mathrm{n}=30$ \\
\hline Session Rating Scale (0-40) & Median (IQR): 37.6 (33.3-40.0) & $\mathrm{n}=30$ \\
\hline
\end{tabular}

\section{Endpoint 2: recruitment and retention}

\begin{tabular}{|c|c|c|}
\hline \multicolumn{3}{|l|}{ Recruitment } \\
\hline \multicolumn{3}{|l|}{ Proportion of those identified who were eligible (\%) } \\
\hline NHS & $64.3(48.0$ to 78.4$)$ & $27 / 42$ \\
\hline Overall & $72.2(58.4$ to 83.5$)$ & $39 / 54$ \\
\hline Criterion 2 met: proportion of eligible participants who consented (\%) & $82.1(66.5$ to 92.5$)$ & $32 / 39$ \\
\hline \multicolumn{3}{|l|}{ Retention } \\
\hline Proportion of withdrawals overall (\%) & $6.3(0.8$ to 20.8$)$ & $2 / 32$ \\
\hline Proportion of withdrawals before randomisation (\%) & 0 (0 to 10.9) & $0 / 32$ \\
\hline \multicolumn{3}{|l|}{ Proportion of withdrawals postrandomisation (\%) } \\
\hline \multicolumn{3}{|c|}{ Criterion 3 met: proportion of participants who were followed up at 6 months postrandomisation (\%) } \\
\hline Intervention arm & 94.1 (71.3 to 99.9$)$ & $16 / 17$ \\
\hline Control arm & 100 (78.2 to 100.0$)$ & $15 / 15$ \\
\hline Overall & 96.9 (83.8 to 99.9$)$ & $31 / 32$ \\
\hline \multicolumn{3}{|l|}{ Endpoint 3: acceptability of outcome measures } \\
\hline Proportion of missing data at the scale level & \multicolumn{2}{|l|}{$0 \%$ missing data } \\
\hline $\begin{array}{l}\text { Criterion } 4 \text { met: proportion of item level missing data, for participants } \\
\text { with mild-moderate aphasia }\end{array}$ & \multicolumn{2}{|c|}{$\begin{array}{l}<0.01 \% \text { for WEMWBS and CPIB; 0\% missing data for GHQ- } \\
12 \text {, FAST, SRS }\end{array}$} \\
\hline $\begin{array}{l}\text { Proportion of missing data at item level for participants with severe } \\
\text { aphasia }\end{array}$ & \multicolumn{2}{|c|}{$\begin{array}{l}0 \% \text { missing data for WEMWBS, CPIB, GHQ-12, FAST; } \\
<0.01 \% \text { for SRS }\end{array}$} \\
\hline $\begin{array}{l}\text { Criterion } 5 \text { met: proportion of missing data at the item level on DISCs } \\
\text { (all participants) }\end{array}$ & \multicolumn{2}{|l|}{$0 \%$ missing data } \\
\hline
\end{tabular}

CPIB, Communicative Participation Item Bank; DISCs, Depression Intensity Scale Circles; FAST, Frenchay Aphasia Screening Test; GHQ-12, General Health Questionnaire-12; NHS, National Health Service; SRS, Session Rating Scale; WEMWBS, Warwick-Edinburgh Mental Well-being Scale.

visits as helpful, felt they had made a personal commitment and wanted to make a success of the project. In addition, a subset of participants found that participating gave them a sense of purpose and made them feel useful.

Primary endpoint 3: acceptability of research procedures and outcome measures

Acceptability of research procedures was evidenced by low attrition rates and the high proportion of assessment visits which took place \pm 14 days of due date $(96.7 \%$ at 3 months, $96.8 \%$ at 6 months and $71.4 \%$ at 9 months). There were seven serious adverse events, all overnight hospital admissions. In addition, there were 21 adverse events, 19 of which related to scoring as 'distressed' on the GHQ-12, the other 2 instances related to safeguarding concerns and a fall. None of the adverse events or serious adverse events were deemed to be associated with participating in the study. There were two instances of unblinding.

There were minimal missing data on clinical outcome measures: no missing data at the scale level; at the item level, no missing data for GHQ-12, DISCs and FAST, and $<0.01 \%$ missing data for the WEMWBS, CPIB and SRS. Real-time notes made by research assistants (RAs) documented concerns about participants with severe 
comprehension difficulties understanding some items from CPIB.

\section{Participant perspectives on research procedures and outcome measures}

Participants were positive about assessment visits as they liked the RAs, who were described as kind, empathetic and genuinely caring. For a subset, the process of being asked questions about their lives by someone concerned and interested was felt to be therapeutic ('helpful, helpful'). The outcome measures were not perceived as burdensome, although some found them tiring, and appreciated being given breaks, as well as support and time to comprehend the questions. The questions were mostly considered appropriate and relevant, although some response options, such as for the DISCs, were felt to lack nuance.

Participants varied in how acceptable they found being allocated to the wait-list group: although many had no preference about group allocation, others were unhappy about waiting 6 months. There were also misunderstandings about the allocation process (eg, that allocation was based on need or managing limited resources).

\section{Primary endpoint 4: feasibility of delivering the intervention by} SLTS

All three trial clinicians considered that it was appropriate for SLTs to deliver the therapy. Initial training (in SFBT, active listening and mental health support) was considered essential, as was monthly SFBT supervision. In addition, clinicians valued being able to access realtime support from either a mental health professional or principal investigator when they had concerns about a participant. They also described benefitting from peer support. More challenging aspects were supporting people in severe distress and involving family where there were family tensions.

A main theme from the interviews with participants with aphasia $(n=30)$ was that the relationship with the therapist was a key ingredient of the therapy. Participants described feeling understood, accepted and valued by their therapist.

\section{Clinical outcomes}

Descriptive statistics are presented in table 3 for clinical outcomes measured at 3, 6 and 9 months postrandomisation, overall and by trial arm. Prespecified primary comparison point was 6 months postrandomisation for WEMWBS. The estimated difference between groups on the WEMWBS at 6 months was 1.03 (95\% CI -3.77 to $5.83)$, with an effect size of 0.10 (95\% CI -0.38 to 0.58$)$; after excluding those with high well-being at baseline, the estimated difference between groups (for $n=20$ ) was 2.39 (95\% CI -3.44 to 8.22 ), with an effect size of $0.36(95 \%$ CI -0.52 to 1.24$)$. The ICC for WEMWBS at 6 months in the intervention arm was 0.03 (95\% CI 0.001 to 1.00$)$. Figure 2 presents standardised treatment effects. In terms of secondary outcomes, the most

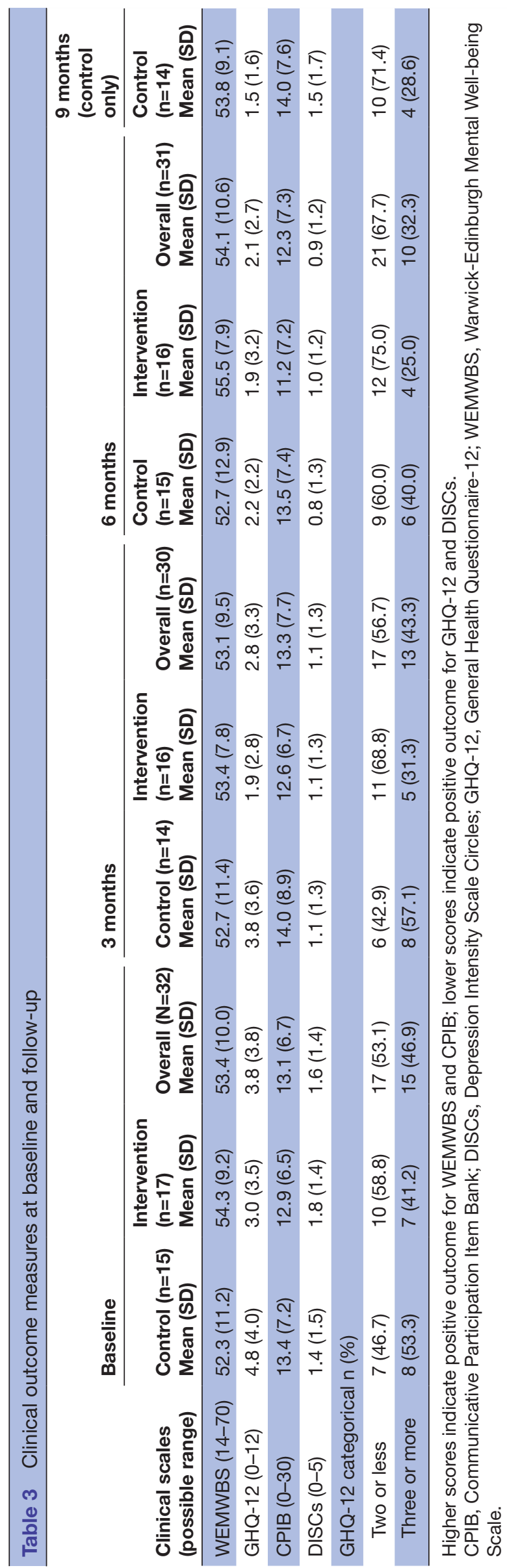




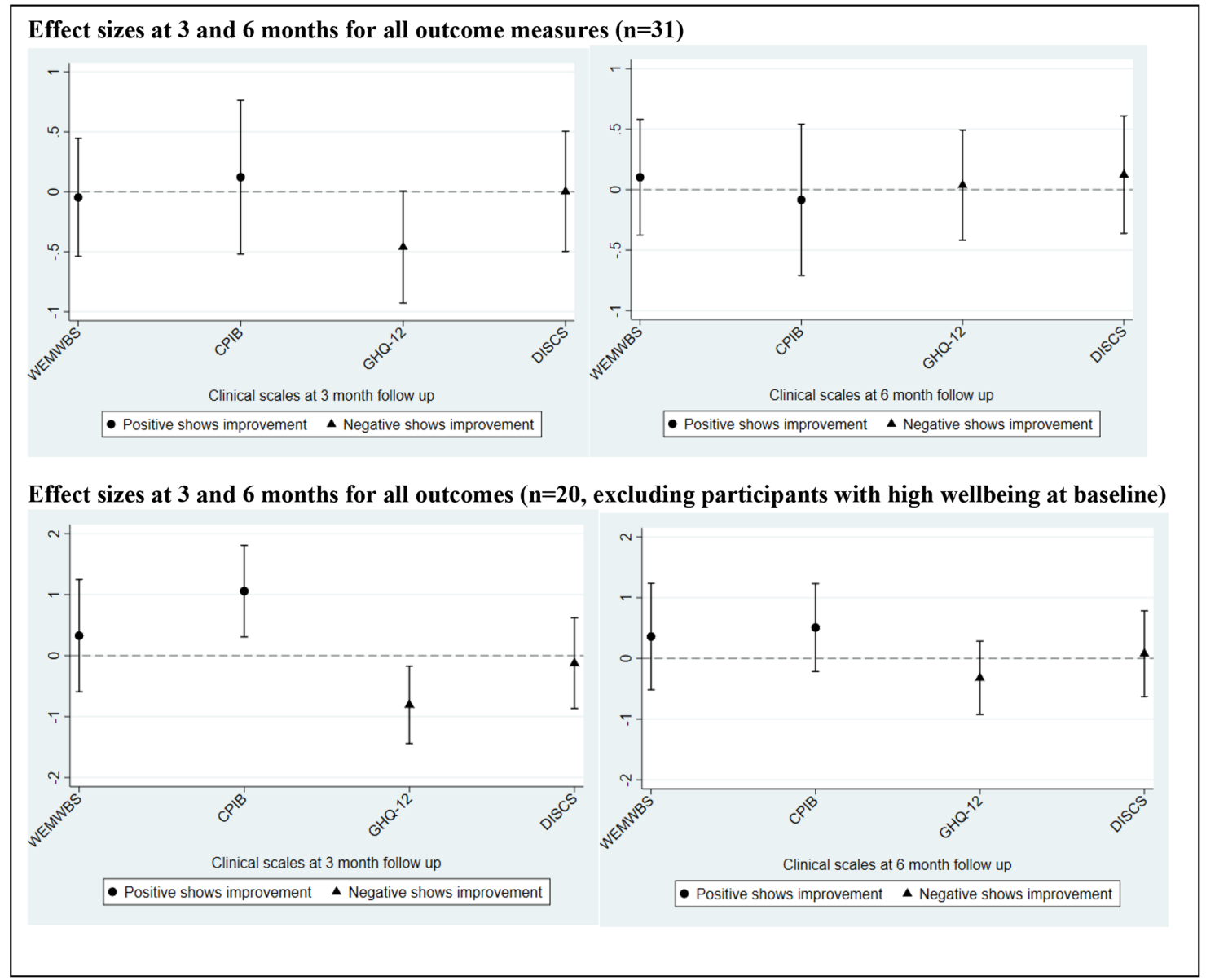

Figure 2 Standardised treatment effects at 3 and 6 months. CPIB, Communicative Participation Item Bank; DISCs, Depression Intensity Scale Circles; GHQ-12, General Health Questionnaire-12; WEMWBS, Warwick Edinburgh Mental Well-being Scale.

marked difference between groups was at 3 months for the GHQ-12, favouring the intervention group, with an effect size of -0.46 (95\% CI -0.93 to 0.01 ). Descriptive statistics were calculated separately for those with mildmoderate versus severe aphasia for all outcome measures: no noteworthy differences were observed. Estimate and effect size differences for all outcome measures at 3 and 6 months are supplied in online supplemental appendix 2.

\section{DISCUSSION}

The SOFIA trial investigated the feasibility of a future definitive trial exploring whether adapted SFBT enhances the well-being of people with poststroke aphasia. All five prespecified progression criteria were satisfied. There was quantitative and qualitative evidence that the approach was acceptable; targets relating to recruitment and retention were met; and there were minimal missing data on outcome measures. It was also feasible for SLTS, with training and supervision, to deliver the intervention.

A common challenge for trials is recruitment, with only a minority of trials recruiting to target and within their prespecified timeline.$^{36}$ Within SOFIA, participants' aphasia meant that conventional forms of communication, such as sending letters, emails or phoning, were often compromised, creating additional challenges. It is therefore encouraging that despite this, it was possible to recruit to target. A review of effective recruitment strategies within primary care research outlined strategies used in SOFIA: straightforward eligibility criteria, nonburdensome research processes, practitioner interest in the research topic and belief in the potential benefits for patients. ${ }^{37}$ Further factors were the perceived benefits for the sites, also found in other research, ${ }^{38}$ and the sense of partnership and collaborative effort. In that sense, the principles of the SOFIA intervention were applied to trial management: working with sites' strengths, building on what was working well, being sensitive to recruiter challenges. The involvement of people with aphasia in designing the trial may also have enabled strong recruitment, ${ }^{39}$ including recruiting participants from diverse backgrounds comparable to the parent stroke population. ${ }^{40}$ Involving different stakeholders may be important when considering how to maintain strong recruitment in a future larger trial. Retention was also strong $(96.9 \%$ at 6 months), comparing favourably to average trial retention rates of $89 \%$ in recent UK Trials. ${ }^{41}$ Excellent completion rates and low attrition may in part reflect that assessment visits were conducted face-to-face by RAs who were also SLTs, and skilled at working with the strengths of people with aphasia (eg, RA leaving a coloured dot on a wall 
chart as the participant's preferred way to remember the next assessment visit).

A promising finding was that people with severe aphasia were enabled to participate. People with severe aphasia have worse quality of life than people with mildmoderate aphasia ${ }^{42}$ yet struggle to access mental health services due to their language difficulties. ${ }^{14}$ SLTs may have an important role in supporting the well-being of those with more profound communication disabilities. The stepped care model suggests that it may be appropriate for stroke healthcare professionals, such as SLTs, to offer brief psychological therapies, so long as they are appropriately trained and supervised. ${ }^{43}$ In line with this model, the current study found that SLTs valued ongoing specialist support, particularly for managing more complex psychological situations (severe distress and family tensions).

As the study was not designed to determine effectiveness and the groups were potentially imbalanced at baseline, caution should be exercised in interpreting differences between groups. There is some modest indication that those with low-moderate well-being at baseline potentially derived more benefit than those with high well-being at baseline, although this may reflect a ceiling effect for participants with high well-being. Nonetheless, there is some triangulatory evidence from the qualitative data to support this pattern. A subgroup of participants with high well-being prior to therapy were described by the SOFIA therapists as 'content in themselves': therapists considered that this group was least likely to make sustained gains due to the therapy. By contrast, therapy was perceived to have made the most difference for participants perceived as 'stuck' or at a 'low ebb' at the start of therapy. This pattern was also found in participant interviews. ${ }^{21}$

Overall, both groups improved on the GHQ-12 mood measure. Although the trial was underpowered, the difference between groups at 3 months appeared to favour the intervention group. This difference was not maintained at 6 months potentially because the intervention group, on average, had achieved normal mood scores by 3 months (maintained at 6 months), while the control group continued to improve. The phenomenon of a wait-list group improving has been observed in other wait-list trials. ${ }^{44}$ Within aphasia research, two studies using the wait-list design reported significant improvements in confidence ${ }^{45}$ and quality of life ${ }^{46}$ during the waiting period for the control group. Anticipation of future therapy may have engendered hope, improving mood. Alternatively, it has been argued that the process of assessing mood within a trial heightens a person's awareness of their low mood, leading them to activate internal and external coping mechanisms. ${ }^{47}$ Within SOFIA, there were anecdotal reports of participants' families rallying around while they waited for the therapy (eg, organising an allotment and increasing contact). It may also relate to the assessment visits which some participants described as therapeutic. While this may have benefitted retention and resonates with a humane approach to outcome measurement, assessment visits were not anticipated to be therapeutic. This arguably deserves further reflection and research. It may be that regular ( 3 monthly) visits from a concerned and skilled listener is a valid therapy in itself.

A strength of the trial was the use of qualitative as well as quantitative data to provide nuanced answers to the research questions. ${ }^{48}$ For example, interviewing local collaborators gave context on successful recruitment processes. A further strength was the measures taken to provide reassurance as to the trustworthiness of the qualitative data analysis (second analyst independently drawing themes from the data and member checking). A weakness of the trial is that although family members were invited to participate in therapy sessions, they were not research participants, and so their views on the therapy could not be captured. A further weakness was that the principal investigator conducted some of the interviews, which could have led to bias. We acknowledge we would have gained a greater understanding of participants' aphasia had we used a full language assessment such as the Western Aphasia Battery-Revised ${ }^{49}$ at baseline rather than a screening measure. Choice of assessments, and how best to measure outcomes, is a challenge within stroke rehabilitation research ${ }^{50}$ : choosing measures that capture reported change and balancing the benefits of gaining additional information against respondent burden. Some of the changes described by participants in the qualitative interviews spanned physical, psychosocial and communication domains (eg, increased independence and confidence talking, improved mood and relationships) ${ }^{21}$ : using a quality of life measure, such as the Stroke and Aphasia Quality of Life Scale- $39,{ }^{51}$ may have picked up these changes. Finally, a main theme from the qualitative interviews was the importance of the therapeutic relationship: a future trial could explore this more fully using the Aphasia and Stroke Therapeutic Alliance Measure, ${ }^{52}$ a measure specifically developed for this client group.

Learning points for a future trial include clarifying more explicitly with potential participants that the intervention is focusing on psychological rather than language work, potentially targeting the intervention at people with low-moderate well-being and considering how best to support clinicians when working with distressed participants. In terms of dosage, there was little variation: almost all participants elected to have all six sessions. From the qualitative interviews with participants, many suggested they would have preferred more individualised dosage, potentially spaced over a longer period of time. ${ }^{21}$ Alternative study designs that address some of the disadvantages of the wait-list design could be considered, such as randomising clusters rather than individuals potentially using a stepped wedge design or randomising individuals to alternative interventions. Future research may also consider whether the therapy can be adapted for online delivery: this may inform both future research as well as clinical implementation. 
The SOFIA trial satisfied all prespecified progression criteria. It is encouraging that trial clinicians were able to adapt SFBT so that it was linguistically accessible and highly acceptable to participants, including people with severe aphasia. These promising feasibility results suggest it is worth exploring clinical effectiveness in a future definitive trial. Since this research shows it is feasible to adapt a language-based psychological intervention, a major clinical implication is that people with aphasia, including severe aphasia, should have equitable access to appropriate mental health support.

\section{Author affiliations}

${ }^{1}$ Centre for Language and Communication Science, City, University of London, London, UK

${ }^{2}$ Division of Rehabilitation and Ageing, University of Nottingham, Nottingham, UK ${ }^{3}$ Department of Biostatistics and Health Informatics, Institute of Psychiatry, Psychology and Neurosciences, King's College London, London, UK ${ }^{4}$ Health Services and Population Research, King's College London, London, UK ${ }^{5}$ Centre for Health Services Research, City, University of London, London, UK

Twitter Sarah Northcott @sarahnorthcott8, Shirley Thomas @DrShirleyThomas, Alan Simpson @cityalan, Rachel Barnard @Rachel_Barnard_and Katerina Hilari @ KaterinaHilari

Acknowledgements The authors are grateful to all the participants, participant identification sites and research assistants. We would like to thank the therapists and local collaborators for their contribution including taking part in interviews. We would also like to thank the SOFIA Aphasia Advisory Group and the Trial Steering Committee for their ongoing support throughout the trial. Finally, we are grateful to Kidge Burns for providing clinical supervision and advising on the therapy, and Professor Richard Hooper for his advice when designing the study. This study was supported by the United Kingdom Clinical Research Collaboration-registered King's Clinical Trials Unit at King's Health Partners, which is partly funded by the NIHR Biomedical Research Centre for Mental Health at South London and Maudsley NHS Foundation Trust and King's College London and the NIHR Evaluation, Trials and Studies Coordinating Centre.

Contributors SN led the project and was principal investigator. KJ conducted the quantitative analysis with SN and SH. SH developed the statistical analysis plan with SN and contributed to the quantitative analysis. SN and RB conducted qualitative interviews and analysed the qualitative data. KH, AS, ST and SH were all named supervisors, contributing to the design and conduct of the study throughout. All authors were involved in interpreting the data, provided critical comment on the manuscript and approved the final version.

Funding This work was supported by the Stroke Association Jack and Averil (Mansfield) Bradley Fellowship Award for Stroke Research (grant number TSA PDF 2016/01). The trial sponsor was City, University of London (Staff/17-18/04), and the study was funded by the Stroke Association (TSAPostdoc2016/01).

Competing interests None declared.

Patient consent for publication Not required.

Ethics approval The study obtained ethical approval from the NHS Health Research Authority Brighton and Sussex Research Ethics Committee (17/L0/1255).

Provenance and peer review Not commissioned; externally peer reviewed.

Data availability statement Data are available upon reasonable request. The study data have not been made available. The participants did not provide consent for datasets to be stored outside of the research team.

Supplemental material This content has been supplied by the author(s). It has not been vetted by BMJ Publishing Group Limited (BMJ) and may not have been peer-reviewed. Any opinions or recommendations discussed are solely those of the author(s) and are not endorsed by BMJ. BMJ disclaims all liability and responsibility arising from any reliance placed on the content. Where the content includes any translated material, BMJ does not warrant the accuracy and reliability of the translations (including but not limited to local regulations, clinical guidelines, terminology, drug names and drug dosages), and is not responsible for any error and/or omissions arising from translation and adaptation or otherwise.
Open access This is an open access article distributed in accordance with the Creative Commons Attribution Non Commercial (CC BY-NC 4.0) license, which permits others to distribute, remix, adapt, build upon this work non-commercially, and license their derivative works on different terms, provided the original work is properly cited, appropriate credit is given, any changes made indicated, and the use is non-commercial. See: http://creativecommons.org/licenses/by-nc/4.0/.

\section{ORCID iDs}

Sarah Northcott http://orcid.org/0000-0001-8229-5452

Shirley Thomas http://orcid.org/0000-0003-0704-9387

Kirsty James http://orcid.org/0000-0002-2657-8148

Alan Simpson http://orcid.org/0000-0003-3286-9846

Shashivadan Hirani http://orcid.org/0000-0002-1577-8806

Rachel Barnard http://orcid.org/0000-0003-4319-9550

Katerina Hilari http://orcid.org/0000-0003-2091-4849

\section{REFERENCES}

1 Flowers HL, Skoretz SA, Silver FL, et al. Poststroke aphasia frequency, recovery, and outcomes: a systematic review and metaanalysis. Arch Phys Med Rehabil 2016;97:e8:2188-201.

2 Northcott S, Marshall J, Hilari K. What factors predict who will have a strong social network following a stroke? J Speech Lang Hear Res 2016;59:772-83.

3 Kauhanen ML, Korpelainen JT, Hiltunen P, et al. Aphasia, depression, and non-verbal cognitive impairment in ischaemic stroke. Cerebrovasc Dis 2000;10:455-61.

4 Baker C, Worrall L, Rose M, et al. 'It was really dark': the experiences and preferences of people with aphasia to manage mood changes and depression. Aphasiology 2020;34:19-46.

5 Shadden B. Aphasia as identity theft: theory and practice. Aphasiology 2005;19:211-23.

6 Hilari K. The impact of stroke: are people with aphasia different to those without? Disabil Rehabil 2011;33:211-8.

7 Hilari K, Northcott S. "Struggling to stay connected": comparing the social relationships of healthy older people and people with stroke and aphasia. Aphasiology 2017;31:674-87.

8 Townend E, Brady M, McLaughlan K. Exclusion and inclusion criteria for people with aphasia in studies of depression after stroke: a systematic review and future recommendations. Neuroepidemiology 2007:29:1-17.

9 Thomas SA, Russell C, Seed R, et al. An evaluation of treatment integrity in a randomized trial of behavioural therapy for low mood in stroke patients with aphasia. Clin Rehabil 2013;27:1097-106.

10 Tarrant M, Carter M, Dean SG, et al. Singing for people with aphasia (spa): results of a pilot feasibility randomised controlled trial of a group singing intervention investigating acceptability and feasibility. BMJ Open 2021;11:e040544.

11 Hilari K, Behn N, James K, et al. Supporting wellbeing through peer-befriending (SUPERB) for people with aphasia: a feasibility randomised controlled trial. Clin Rehabil 2021;35:1151-63.

12 Baker C, Worrall L, Rose M, et al. A systematic review of rehabilitation interventions to prevent and treat depression in poststroke aphasia. Disabil Rehabil 2018;40:1870-92.

13 Baker C, Worrall L, Rose M, et al. Stroke health professionals' management of depression after post-stroke aphasia: a qualitative study. Disabil Rehabil 2019:1-12.

14 Northcott S, Simpson A, Moss B, et al. Supporting people with aphasia to 'settle into a new way to be': speech and language therapists' views on providing psychosocial support. Int J Lang Commun Disord 2018;53:16-29.

15 Burns K. Focus on solutions: a health professional's guide. 2016. 2 edn. Gloucester: Solutions Books, 2016.

16 Carrick H, Randle-Phillips C. Solution-focused approaches in the context of people with intellectual disabilities: a critical review. $J$ Ment Health Res Intellect Disabil 2018;11:30-53.

17 Zhang A, Franklin C, Currin-McCulloch J, et al. The effectiveness of strength-based, solution-focused brief therapy in medical settings: a systematic review and meta-analysis of randomized controlled trials. $J$ Behav Med 2018;41:139-51.

18 Wichowicz HM, Puchalska L, Rybak-Korneluk AM, et al. Application of Solution-Focused brief therapy (SFBT) in individuals after stroke. Brain Inj 2017;31:1507-12.

19 Northcott S, Burns K, Simpson A, et al. 'Living with aphasia the best way I can': a feasibility study exploring Solution-Focused brief therapy for people with aphasia. Folia Phoniatr Logop 2015;67:156-67. 
20 Northcott S, Simpson A, Thomas SA, et al. Solution focused brief therapy in post-stroke aphasia (Sofia trial): protocol for a feasibility randomised controlled trial. AMRC Open Res 2019;1:11.

21 Northcott S, Simpson A, Thomas S, et al. "Now I Am Myself": Exploring How People With Poststroke Aphasia Experienced Solution-Focused Brief Therapy Within the SOFIA Trial. Qual Health Res 2021;0:104973232110202.

22 Eldridge SM, Chan CL, Campbell MJ, et al. Consort 2010 statement: extension to randomised pilot and feasibility trials. Pilot Feasibility Stud 2016;2:64.

23 O'Brien BC, Harris IB, Beckman TJ, et al. Standards for reporting qualitative research: a synthesis of recommendations. Acad Med 2014;89:1245-51.

24 de Shazer S. Clues: investigating solutions in brief therapy. New York: W W Norton, 1988.

25 Ratner H, George E, Iveson C. Solution Focused Brief Therapy: 100 key points and techniques.. In: Dryden W. Hove: Routledge, 2012.

26 Enderby P, Wood V, Wade D. Frenchay aphasia screening test. Windsor UK: NFEW-Nelson, 1987.

27 NHS Health Scotland. Short Warwick Edinburgh mental well being scale, 2007. University of Warwick University of Edinburgh

28 Goldberg D. The detection of psychiatric illness by questionnaire. London, UK: Oxford University Press, 1972.

29 Turner-Stokes L, Kalmus M, Hirani D, et al. The depression intensity scale circles (DISCS): a first evaluation of a simple assessment tool for depression in the context of brain injury. $J$ Neurol Neurosurg Psychiatry 2005;76:1273-8.

30 Baylor C, Yorkston K, Eadie T, et al. The communicative participation item bank (CPIB): item bank calibration and development of a disorder-generic short form. $J$ Speech Lang Hear Res 2013;56:1190-208.

31 Duncan BL, Miller SD, Sparks JA. The Session Rating Scale: Preliminary psychometric properties of a "working" alliance measure. Journal of brief Therapy 2003;3:3-12.

32 Lancaster GA, Dodd S, Williamson PR. Design and analysis of pilot studies: recommendations for good practice. J Eval Clin Pract 2004;10:307-12.

33 Julious SA. Sample size of 12 per group rule of thumb for a pilot study. Pharm Stat 2005;4:287-91.

$34 \mathrm{Sim}$ J, Lewis $\mathrm{M}$. The size of a pilot study for a clinical trial should be calculated in relation to considerations of precision and efficiency. $J$ Clin Epidemiol 2012;65:301-8.

35 Ritchie J, Spencer L. Qualitative data analysis for applied policy research. In: Bryman A, Burgess R, eds. Analysing qualitative data. London: Routledge, 1994.

36 Sully BGO, Julious SA, Nicholl J. A reinvestigation of recruitment to randomised, controlled, multicenter trials: a review of trials funded by two UK funding agencies. Trials 2013;14:166.
37 Ngune I, Jiwa M, Dadich A, et al. Effective recruitment strategies in primary care research: a systematic review. Qual Prim Care 2012;20:115-23.

38 Vluggen S, Hoving C, Vonken L, et al. Exploring factors influencing recruitment results of nurses recruiting diabetes patients for a randomized controlled trial. Clin Trials 2020;17:448-458:448-58.

39 Witham MD, Anderson E, Carroll C, et al. Developing a roadmap to improve trial delivery for under-served groups: results from a UK multi-stakeholder process. Trials 2020;21:694.

40 Wang Y, Rudd AG, Wolfe CDA. Age and ethnic disparities in incidence of stroke over time: the South London stroke register. Stroke 2013;44:3298-304.

41 Walters SJ, Bonacho Dos Anjos Henriques-Cadby I, Bortolami $\mathrm{O}$, et al. Recruitment and retention of participants in randomised controlled trials: a review of trials funded and published by the United Kingdom health technology assessment programme. BMJ Open 2017;7:e015276.

42 Hilari K, Byng S. Health-related quality of life in people with severe aphasia. Int J Lang Commun Disord 2009;44:193-205.

43 Improvement NHS. Psychological care after stroke: improving stroke services for people with cognitive and mood disorders, 2011. Available: http://www.nice.org.uk/

44 Hesser H, Weise C, Rief W, et al. The effect of waiting: a metaanalysis of wait-list control groups in trials for tinnitus distress. $J$ Psychosom Res 2011;70:378-84.

45 Marshall J, Booth T, Devane N, et al. Evaluating the benefits of aphasia intervention delivered in virtual reality: results of a QuasiRandomised study. PLoS One 2016;11:e0160381.

46 Breitenstein C, Grewe T, Flöel A, et al. Intensive speech and language therapy in patients with chronic aphasia after stroke: a randomised, open-label, blinded-endpoint, controlled trial in a health-care setting. Lancet 2017;389:1528-38.

47 Arrindell WA. Changes in waiting-list patients over time: data on some commonly-used measures. beware! Behav Res Ther 2001;39:1227-47.

48 O'Cathain A, Thomas KJ, Drabble SJ, et al. What can qualitative research do for randomised controlled trials? A systematic mapping review. BMJ Open 2013;3:e002889.

49 Kertesz A. Western aphasia battery-revised (WAB-R). Hoboken: NJ: Pearson, 2006

50 Dean SG, Poltawski L, Forster A, et al. Community-Based rehabilitation training after stroke: results of a pilot randomised controlled trial (retrain) investigating acceptability and feasibility. BMJ Open 2018;8:e018409.

51 Hilari K, Lamping DL, Smith SC, et al. Psychometric properties of the stroke and aphasia quality of life scale (SAQOL-39) in a generic stroke population. Clin Rehabil 2009;23:544-57.

52 Lawton M, Conroy P, Sage K, et al. Aphasia and stroke therapeutic alliance measure (A-STAM): development and preliminary psychometric evaluation. Int J Speech Lang Pathol 2019;21:459-69. 